\title{
PYRIDINE-NUCLEOTIDE COENZYMES IN BULL AND RABBIT SPERMATOZOA
}

\author{
M. BISTOCGHI, G. D'ALESSIO AND E. LEONE \\ Istituto di Chimica Biologica, Università di Perugia, e Impresa di Enzimologia del \\ Consiglio Nazionale delle Ricerche, Italy
}

(Received 3rd Fuly 1967)

\begin{abstract}
Summary. The content of the two pyridine-nucleotide coenzymes, namely nicotinamide adenine dinucleotide (NAD) and nicotinamide adenine dinucleotide phosphate (NADP), has been determined in bull and rabbit spermatozoa, using the enzymic-fluorimetric procedure of Bassham, Birt, Hems \& Loening (1959). It has been found necessary to wash spermatozoa thoroughly before extraction, so as to remove all traces of seminal plasma enzymes which otherwise would have degraded the coenzymes in the course of the analytical procedure. In epididymal spermatozoa a markedly higher content of pyridine-nucleotide coenzymes has been found, compared to that in ejaculated spermatozoa. Incubation experiments distinctly indicated a lability of the coenzymes in both types of spermatozoa. It has also been shown that a very short ( 1 min) incubation of epididymal spermatozoa with seminal plasma causes a definite decrease in the coenzyme content.
\end{abstract}

\section{INTRODUCTION}

Mammalian spermatozoa are known to possess both glycolytic and respiratory activity (Mann, 1964), but data are scarce on the levels of pyridine-nucleotide coenzymes (PNC) in these cells. Winberg (1941) has been the first to detect the presence of NAD in bird spermatozoa. More recently, Gonse (1962) used a spectrophotometric procedure to evaluate the relative proportions of cytochromes, flavoproteins and PNG in bull spermatozoa. In that study, however, PNC were determined as a whole, that is as the sum of NAD and NADP. Nor did that technique permit the separate determination of the oxidized forms (NAD, NADP) and the reduced forms (NADH, NADPH) of the two coenzymes. In our study, an attempt was made to investigate not merely the PNC as a whole, but to determine separately, by the use of sensitive and specific analytical methods, the contents of NAD, NADH, NADP and NADPH in bull spermatozoa (Bistocchi \& D'Alessio, 1964; Bistocchi, D'Alessio \& Leone, 1964). Our interest has now been extended to the determination of PNG levels in epididymal bull spermatozoa, which are known to differ both morphologically and functionally from ejaculated spermatozoa. Such research has appeared worthwhile in view of the largely obscure nature of the biochemical changes 
associated with the ripening of spermatozoa in the epididymis. We have also carried out a number of incubation experiments with both epididymal and ejaculated spermatozoa, in order to assess the degree of the stability of the coenzymes in the living cells (preliminary communication, Bistocchi, D'Alessio \& Leone, 1965). In addition to bull spermatozoa, those of the rabbit, both epididymal and ejaculated, have also been analysed.

\section{MATERIALS AND METHODS}

\section{Collection and washing of spermatozoa}

Semen was collected by means of an artificial vagina. Analyses of bull semen started 20 to 30 min after ejaculation; rabbit semen, pooled from three or four animals, was used immediately. Spermatozoa were separated from plasma by centrifugation at $1085 \mathrm{~g}$ for $15 \mathrm{~min}$, and washed three times with Ringerphosphate solution (RP) containing $0.5 \mathrm{~g}$ of fructose $/ 100 \mathrm{ml}$, according to Mann (1964); each time three volumes of RP were used for washing. Fructose was omitted in the final suspension since the presence of the sugar interfered with the fluorimetric procedure. The temperature during the whole analysis was kept between $16^{\circ}$ and $18^{\circ} \mathrm{C}$. The spermatozoa were extracted for PNC determinations immediately after washing and resuspension, sperm motility having been checked in the presence of fructose at $37^{\circ} \mathrm{C}$.

Epididymal semen was obtained by expression from the cauda, within $1 \mathrm{hr}$ of the slaughter of the bull, and immediately from rabbits. It was diluted with $\mathrm{RP}$, to obtain a sperm concentration of $10^{9} / \mathrm{ml}$. Separation and washing of epididymal spermatozoa was carried out as described above, except that no fructose was added to the diluent.

Seminal plasma and epididymal plasma obtained by the first centrifugation of semen, were centrifuged again for $15 \mathrm{~min}$ at $20,000 \mathrm{~g}$ to remove cellular debris, and either used immediately or kept at $-15^{\circ} \mathrm{C}$.

\section{Analytical methods}

For routine analyses the method of Bassham et al. (1959) has been followed; this depends on fluorimetric measurements made on samples of acid or alkaline extracts of spermatozoa treated with the specific enzymes, namely alcohol dehydrogenase for NAD, and glucose 6-phosphate dehydrogenase for NADP, in the presence of the respective substrates. A model 110 Turner fluorimeter has been used, equipped with one primary (No. 7-60) and two secondary (Nos. 3 and 48) filters. As a second, independent method of determination, the procedure described by Villee (1962) has also been frequently applied to the analysis of PNC in the incubation experiments; this is based on the spectrophotometric measurement of the rate of decrease of the optical density at $600 \mathrm{~m} \mu$, due to reduction of 2,6-dichlorophenol indophenol.

Fructolysis was determined according to Mann (1948).

Alcohol dehydrogenase (ADH) and glucose 6-phosphate dehydrogenase were commercial products (Boehringer). All coenzymes used as standards were Sigma products, and their purity was checked by spectrophotometry and paper chromatography. 


\section{RESULTS}

The effect of seminal plasma and epididymal plasma on $P \mathcal{N C}$

Preliminary PNC determinations carried out either in whole ejaculated bull semen or seminal plasma or in epididymal semen gave negative results. It is only when the spermatozoa had been thoroughly washed that positive results were obtained. Incubation of bull seminal plasma or of wash fluids with NAD, followed by NAD assay by the cyanide reaction (Ciotti \& Kaplan, 1957), showed that some of the well-known high NADase activity of seminal plasma (Leone \& Bonaduce, 1959) was carried into the first and second wash fluid. At least three washings of spermatozoa were required to remove NADase activity completely. NADH-splitting activity, previously described by Wheat, Krick \& Brownlee (1960), was also still detectable in the second wash fluid, as measured by the ADH reaction (Ciotti \& Kaplan, 1957). Not only seminal plasma, but epididymal plasma as well, showed PNG-splitting activity directed against both NAD and NADH. However, since with this material negative results were obtained by the cyanide reaction, but positive results by the ADH test, it may be concluded that PNG-pyrophosphatase is mainly responsible for the PNC-splitting activity of epididymal plasma; the NADase activity is negligible or altogether absent (Bistocchi \& Fettuccia, 1966).

TABLE 1

RECOVERY EXPERIMENTS IN WHICH PNC WERE ADDED TO WASHED BULL SPERMATOZOA JUST BEFORE THE EXTRACTION; THE PNG CONTENT IS EXPRESSED IN m $\mu$ moles $/ 10^{9}$ SPERMATOZOA; IT WAS DETERMINED BY THE PROCEDURE of BASSHAM et al. (1959)

\begin{tabular}{|c|c|c|c|c|c|c|}
\hline \multirow[b]{2}{*}{ Coenzyme } & \multicolumn{3}{|c|}{ Ejaculated spermatozoa } & \multicolumn{3}{|c|}{ Epididymal spermatozoa } \\
\hline & Endogenous & $\begin{array}{c}\text { Endogenous } \\
\text { plus } \\
1 \text { mumole } \\
\text { exogenous } P N C\end{array}$ & $\begin{array}{c}\text { Recovery of } \\
\text { exogenous PNC } \\
(\%)\end{array}$ & Endogenous & $\begin{array}{c}\text { Endogenous } \\
\text { plus } \\
1 \text { mumole } \\
\text { exogenous } P \mathcal{N} C\end{array}$ & $\begin{array}{c}\text { Recovery of } \\
\text { exogenous PNC } \\
(\%)\end{array}$ \\
\hline $\begin{array}{l}\text { NAD } \\
\text { NADH } \\
\text { NADP } \\
\text { NADPH }\end{array}$ & $\begin{array}{l}5 \cdot 09 \\
0.80 \\
0 \cdot 62 \\
0.74\end{array}$ & $\begin{array}{l}6.07 \\
1 \cdot 72 \\
1 \cdot 48 \\
1 \cdot 67\end{array}$ & $\begin{array}{l}98 \\
92 \\
86 \\
93\end{array}$ & $\begin{array}{r}12 \cdot 15 \\
0 \cdot 90 \\
3 \cdot 50 \\
1 \cdot 20\end{array}$ & $\begin{array}{r}13 \cdot 18 \\
1 \cdot 84 \\
4 \cdot 40 \\
2 \cdot 14\end{array}$ & $\begin{array}{r}103 \\
94 \\
90 \\
94\end{array}$ \\
\hline
\end{tabular}

Similar results were also obtained with rabbit seminal plasma, and it was therefore decided to perform always at least three washings before any PNC determination in either ejaculated or epididymal spermatozoa. Under such conditions, recovery of added ('exogenous') coenzymes was satisfactory, both as regards their oxidized and reduced forms, in ejaculated as well as epididymal spermatozoa (Table 1).

Content and distribution of PNC in washed ejaculated and epididymal spermatozoa

A series of determinations was carried out on both bull and rabbit spermatozoa, using the fluorimetric method (Bassham et al., 1959); total oxidized and total reduced coenzymes were estimated, without differentiating, however, between NAD and NADP; the results appear in Table 2, from which it can be seen that the concentration of oxidized PNG is about three times higher in 
epididymal than in ejaculated spermatozoa; the concentration of reduced PNC is also somewhat higher in epididymal than ejaculated spermatozoa (ratio of about $1 \cdot 5: 1$ ).

TABLE 2

THE CONTENT OF TOTAL OXIDIZED AND TOTAL REDUCED PNG IN WASHED BULL AND RABBIT SPERMATOZOA (m $\mu$ moles $/ 10^{9}$ SPERMATOZOA), DETERMINED ACCORDING TO BASSHAM et al. (1959)

\begin{tabular}{l|c|c|c}
\hline & & Ejaculated spermatozoa & Epididymal spermatozoa \\
\hline Bull & NAD+NADP & $6 \cdot 75 \pm 0 \cdot 20$ & $24 \cdot 51 \pm 1 \cdot 26$ \\
(eighteen experiments) & & $(5 \cdot 20-9 \cdot 55)$ & $(16.90-40 \cdot 80)$ \\
& NADH+ NADPH & $2 \cdot 38 \pm 0 \cdot 13$ & $3 \cdot 16 \pm 0 \cdot 23$ \\
& & $(1 \cdot 47-3 \cdot 35)$ & $(1 \cdot 10-4 \cdot 50)$ \\
\hline Rabbit & NAD+NADP & $11 \cdot 00 \pm 1 \cdot 33$ & $27 \cdot 33 \pm 1 \cdot 05$ \\
(six experiments) & NADH+NADPH & $(6 \cdot 9-14 \cdot 0)$ & $(23 \cdot 0-30.0)$ \\
& & $(1 \cdot 67 \pm 0 \cdot 13$ & $2 \cdot 50 \pm 0.21$ \\
& & $(1 \cdot 8-3 \cdot 1)$ \\
\hline
\end{tabular}

Values represent means $\pm \mathrm{SEM}$, with ranges in parentheses.

Next, determinations were performed in such a way as to measure separately the oxidized and reduced forms of the coenzymes. Such determinations could be made, however, only with bull sperm since rabbit semen, either ejaculated or epididymal, had too low density and volume to allow a complete analysis. A distinctly higher concentration of oxidized coenzymes was observed in the epididymal than in ejaculated spermatozoa (Table 3). A smaller, but nevertheless significant difference $(P<0.001)$ was observed as regards the reduced forms of coenzymes.

TABLE 3

THE CONTENT OF VARIOUS FORMS OF PNG IN BULL SPERMATOZOA ( $\mathrm{m} \mu$ moles $/ 10^{9}$ SPERMATOZOA), DETERMINED ACCORDING TO BASSHAM et al. (1959)

\begin{tabular}{l|c|c}
\hline & Ejaculated spermatozoa & Epididymal spermatozoa \\
\cline { 2 - 3 } NAD & $5 \cdot 54 \pm 0.23$ & $17 \cdot 47 \pm 0.81$ \\
& $(4 \cdot 78-6 \cdot 10)$ & $(15 \cdot 10-20 \cdot 00)$ \\
NADH & $0.83 \pm 0.05$ & $1 \cdot 35 \pm 0.06$ \\
& $(0 \cdot 61-1 \cdot 10)$ & $(1 \cdot 20-1 \cdot 60)$ \\
NADP & $0.94 \pm 0.09$ & $5 \cdot 45 \pm 0.32$ \\
& $(0 \cdot 70-1 \cdot 25)$ & $(4 \cdot 60-6.60)$ \\
NADPH & $1 \cdot 46 \pm 0.08$ & $2 \cdot 50 \pm 0.09$ \\
& $(1 \cdot 20-1 \cdot 70)$ & $(2 \cdot 20-2 \cdot 70)$ \\
\hline
\end{tabular}

Values represent the means of six determinations \pm SEM, with ranges in parentheses.

Owing to the time interval between the collection of bull semen at the insemination centre and the start of analytical procedures in the laboratory, the possibility had to be taken into account that, at ejaculation, the PNG content of spermatozoa was higher than that subsequently recorded in the laboratory. An 
experiment was, therefore, done in which centrifugation and washing of spermatozoa were carried out immediately after collection of the ejaculate. However, the results obtained in this way did not differ from the average values recorded in Table 2. The concentrations were: $5 \mathrm{~m} \mu$ moles of oxidized, and $1.26 \mathrm{~m} \mu$ moles of reduced, $\mathrm{PNC} / 10^{9}$ spermatozoa.

PNC determinations in epididymal spermatozoa were usually made using the material obtained by expression of the cauda epididymidis as a whole, so that spermatozoa of varying degrees of ripeness may have been present. Nevertheless, in order to investigate the possibility that spermatozoa from different parts of the cauda, and, therefore, presumably at different stages of maturation, may also have a different PNC content, an experiment was performed in which the content of total oxidized PNC was determined in spermatozoa obtained from the anterior, middle and posterior portion of the cauda, respectively. As can be seen from Table 4, there was no marked difference in the PNC content of the spermatozoa obtained from these three parts of the cauda.

\section{TABle 4}

THE CONTENT OF OXIDIZED PNC IN SPERMATOZOA FROM DIFFERENT PORTIONS OF CAUDA EPIDIDYMIDIS $\left(\mathrm{m} \mu\right.$ moles $/ 10^{9}$ SPERMATOZOA $)$, DETERMINED AGGORDING TO BASSHAM et al. (1959)

\begin{tabular}{c|c|c|c}
\hline \multirow{3}{*}{ Experiment } & \multicolumn{3}{|c}{ Portion of cauda epididymidis } \\
\cline { 2 - 4 } & $\begin{array}{c}\text { Nearest } \\
\text { to testis }\end{array}$ & Middle & $\begin{array}{c}\text { Nearest } \\
\text { to vas }\end{array}$ \\
\hline 1 & $19 \cdot 8$ & $18 \cdot 2$ & - \\
2 & 28.0 & 28.0 & 20 \\
\hline
\end{tabular}

Incubation experiments with epididymal and ejaculated spermatozoa

In order to explain the difference between the PNG content of epididyrnal ard ejaculated spermatozoa, two possibilities were envisaged: first, that owing to the lability of the epididymal pyridine-nucleotide coenzymes, their concentration decreases as soon as the spermatozoa have left the cauda epididymidis; and secondly, that the seminal plasma contains a factor, presumably of enzymic nature, which is responsible for the breakdown of the sperm-PNC.

To test the first hypothesis, experiments were done, in which the PNC concentration was compared, using washed spermatozoa obtained from fresh and incubated bull semen, either epididymal or ejaculated. As can be seen from Text-fig. 1 , in ejaculated spermatozoa a steady decline of the PNC content has taken place when whole semen was incubated anaerobically at $37^{\circ} \mathrm{G}$, this in spite of the fact that neither fructolysis nor motility appeared to be significantly affected. Essentially the same result has been obtained with epididymal spermatozoa. Of additional interest has been the observation recorded in Textfig. 2, that it makes little difference whether semen (ejaculated or epididymal) is incubated before washing the spermatozoa, or whether the spermatozoa are 


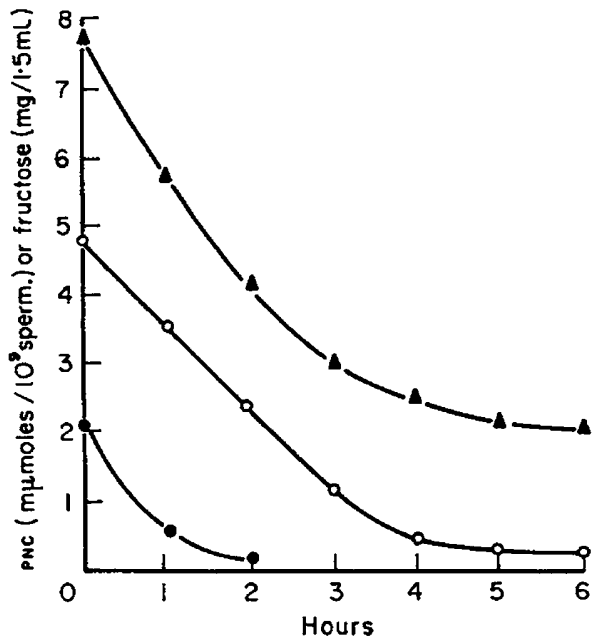

TeXT-FIG. 1. The decrease of the PNC and fructose concentration in bull semen incubated at $37^{\circ} \mathrm{C}$. Fresh bull semen was diluted with Na phosphate buffer, $\left.0.25 \mathrm{M}, \mathrm{pH} 7 \cdot 4,2: 1 \mathrm{v} / \mathrm{v}\right)$, incubated in small test tubes, $0.5 \mathrm{~cm}$ internal diameter, $7.0 \mathrm{~cm}$ length, almost completely filled. At indicated time intervals samples were taken, centrifuged, washed (see under 'Materials and Methods') and PNC (total oxidized and total reduced) determined in washed spermatozoa. Fructose was determined in the whole, diluted semen. $\Delta$, Total oxidized PNC; $O$, fructose; 9 , total reduced PNC.

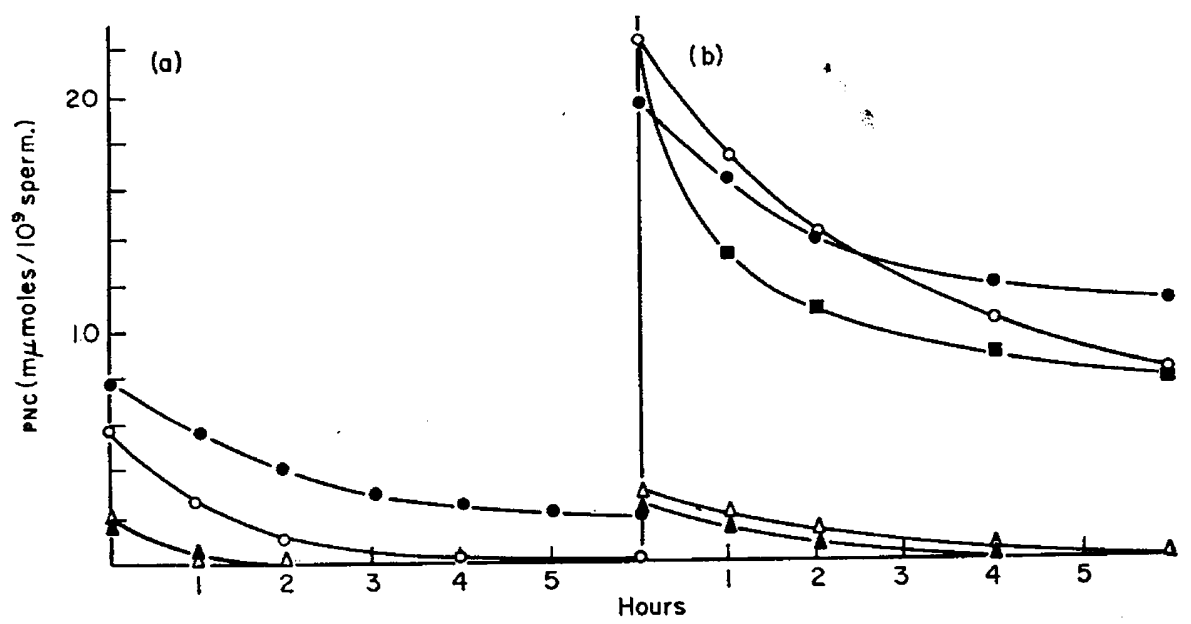

TEXT-FIG. 2. The decrease of PNC concentration in bull spermatozoa incubated at $37^{\circ} \mathrm{C}$. Fresh ejaculated bull semen (a) was diluted as described in Text-fig. 1. Epididymal semen (b) was diluted with RP so as to obtain a sperm concentration of about $0.7 \times 10^{9}$ spermatozoa/ml and either incubated as such or the spermatozoa washed and made up into a suspension with RP $\left(0.7 \times 10^{9}\right.$ spermatozoa $\left./ \mathrm{ml}\right)$ before incubation. Incubation was performed as in Text-fig. 1, samples being taken at the various time intervals for PNC determinations: $\bullet$, whole semen, oxidized PNC; $\Lambda$, whole semen, reduced PNG; $O$, washed spermatozoa, RP suspension, oxidized PNC; $\square$, washed spermatozoa, RP suspension, oxidized PNC plus fructose $(5 \mathrm{mg} / \mathrm{ml}) ; \Delta$, washed spermatozoa, RP suspension, reduced PNG. 
first separated from semen by centrifugation and washing and incubated afterwards.

In order to explore the second possibility, namely, the existence of a degrading effect of seminal plasma on the PNC content of spermatozoa, experiments have been performed on mixtures consisting of epididymal spermatozoa and ejaculated seminal plasma, which were incubated for only $1 \mathrm{~min}$ at $37^{\circ} \mathrm{C}$ before centrifugation and washing of spermatozoa.

These experiments, as presented in Table 5, were divided into three groups:

TABLE 5

THE CONTENT OF TOTAL OXIDIZED (PN) AND TOTAL REDUCED (PNC) PYRIDINE-NUCLEOTIDE GOENZYMES IN EPIDIDYMAL SPERMATOZOA, AFTER 1 min INGUBATION WITH EITHER RINGER-PHOSPHATE OR SEMINAL PLASMA (m $\mu$ moles $/ 10^{9}$ SPERMATOZOA), DETERMINED ACGORDING TO BASSHAM et al. (1959)

\begin{tabular}{c|cc|cc}
\hline & \multicolumn{4}{|c}{ Incubation medium } \\
\cline { 2 - 5 } & \multicolumn{3}{|c|}{ Ringer-phosphate } & \multicolumn{2}{c}{ Seminal plasma } \\
\cline { 2 - 5 } & $P \mathcal{N}$ & $P \mathcal{N C}$ & $P \mathcal{N}$ & $P \mathcal{N C}$ \\
\hline Group A & & & & \\
1 & 23.0 & 2.1 & 13.7 & 2.2 \\
2 & 26.5 & - & 19.3 & - \\
3 & 27.2 & - & 16.5 & - \\
4 & 21.6 & 4.2 & 18.5 & 2.2 \\
5 & 28.0 & 2.6 & 15.6 & 2.0 \\
6 & 25.0 & 3.1 & 17.5 & 2.5 \\
Group B & & & & \\
7 & 17.8 & 1.8 & 1.0 & 2.0 \\
8 & 19.7 & 2.6 & 2.1 & 2.5 \\
Group C & & & & \\
9 & 10.2 & 1.9 & 10.4 & 1.8 \\
10 & 12.2 & - & 11.9 & - \\
11 & 13.5 & - & 14.0 & - \\
12 & 14.1 & 2.0 & 13.0 & 2.1 \\
\hline
\end{tabular}

See text for definition of groups A, B and $\mathrm{C}$.

A, B and C. Group A refers to epididymal spermatozoa obtained from excised epididymides within $1 \mathrm{hr}$ of slaughter, which exhibited good motility when examined after the addition of fresh seminal plasma. Group B refers to epididymal spermatozoa which were obtained in a similar manner, but were mixed with 'aged' seminal plasma, 'aged' by being kept frozen for a period of several weeks; under such conditions spermatozoa showed poor motility and had a tendency to agglutinate. Group $\mathrm{C}$ refers to epididymal spermatozoa obtained from epididymides which had been used only 3 to $4 \mathrm{hr}$ after slaughter; these spermatozoa, although showing good motility, were characterized by a low level of PNC, as determined in suspensions of spermatozoa and Ringer solution (instead of seminal plasma).

It can be seen from Table 5 that, in group A, a definite decrease of PNC resulted from a brief incubation of epididymal spermatozoa with ejaculated, 
fresh seminal plasma. An even larger decrease occurred in group B, but no appreciable loss was observed in group $\mathrm{C}$. If one takes group $\mathrm{A}$ as one which is nearest to physiological conditions associated with the process of ejaculation, it becomes clear that the addition of seminal plasma to epididymal spermatozoa causes within $1 \mathrm{~min}\left(\right.$ at $37^{\circ} \mathrm{C}$ ) a definite decrease of PNC; but even so, the low values which are thus reached are not as low as those which are characteristic of the PNC content in ejaculated spermatozoa.

\section{DISCUSSION}

The finding that epididymal spermatozoa possess a higher concentration of PNC than ejaculated spermatozoa, raises some interesting questions. The experiments in which epididymal spermatozoa were incubated, either in the presence or in the absence of the epididymal plasma, with or without added fructose, show that a marked decrease of the sperm PNC content occurs as a result of such incubations. Essentially the same phenomenon has been observed in ejaculated spermatozoa, which leads us to conclude that the pyridine nucleotide coenzymes, which exist in the spermatozoa, are of a rather labile nature and can be easily depleted during sperm incubation. Even more important, however, is the finding that when epididymal spermatozoa are brought into contact with seminal plasma for as little as $1 \mathrm{~min}$ and then centrifuged and washed, their PNC content is strongly depressed. It looks, therefore, as if the seminal plasma contains a factor which is responsible for the marked decrease of PNG, when epididymal spermatozoa come into contact with it. This is not surprising, as it is well known that seminal plasma is very rich in proteolytic and hydrolytic enzymes (Mann, 1964), as well as in nucleolytic enzymes (Leone \& Santoianni, 1959). It would appear that some degree of alteration occurs as soon as the spermatozoa which left the epididymis come in contact, immediately before ejaculation, with the secretions from the accessory glands. In the bull, the seminal vesicle secretion may be, in this respect, particularly important since, as shown by Bennett \& Dott (1966), the dilution of bull epididymal spermatozoa with seminal plasma causes an immediate decrease of the impedance change frequency; this decrease does not occur, however, when the whole seminal plasma is replaced by a seminal fluid fraction obtained by the electrical stimulation method, which contains certain accessory secretions but not the one produced in the seminal vesicles. There may, of course, be other reasons for the peculiar fragility of pyridine-nucleotide coenzymes within the sperm cells. This phenomenon may, for example, be related to sperm ripening, and the 'dehydration' which accompanies this process. There is the possibility that some sort of 'compartmentalization' of PNC occurs inside the spermatozoon and that, for instance, one part of these coenzymes is tightly bound to cellular structures (e.g. mitochondria or sperm membrane), whereas another part occurs in a free, and therefore more diffusible and extractable state. It is conceivable that the process of protoplasmic shrinkage or 'dehydration' could lead to a loss of the 'diffusible' coenzymes. In that connection, it is worth noting that epididymal spermatozoa as such may exhibit an intrinsic lability as regards their PNC content, even if they had no contact with seminal plasma (see group $\mathrm{C}$ results, 
Table 5). If, namely, epididymal semen is obtained 3 to $4 \mathrm{hr}$ after a bull had been slaughtered the initial values of PNC in epididymal spermatozoa are significantly lower than from epididymides immediately after slaughter. Furthermore, 'aged' seminal plasma, that is one which had been kept in coldstorage for a long time, has a stronger inactivating effect on spermatozoa than fresh seminal plasma, possibly as a result of greater cell damage, and consequently, higher rate of leakage.

\section{ACKNOWLEDGMENTS}

This investigation has been supported in part by the Population Council, New York (Grant M-64.116). We also gratefully acknowledge the help from the Director of the Centro Tori 'Spagnoli' of Perugia, Dr A. Chiacchierini, for the samples of bull semen.

\section{REFERENCES}

Bassham, J. A., Birt, L. M., Hems, R. \& Loening, U. E. (1959) Determination of the reduced and oxidized pyridine nucleotides in animal tissues. Biochem. 7. 73, 491.

BennetT, J. P. \& Dotr, H. M. (1966) An effect of bovine seminal plasma on the impedance change frequency of epididymal spermatozoa collected from the living bull. F. Reprod. Fert. 12, 327.

Bistocchi, M. \& D'Alessio, G. (1964) Coenzimi piridinici in spermatozoi. Boll. Soc. ital. Biol. sper. 40, 2054.

Bistocchi, M., D’Alessio, G. \& Leone, E. (1964) Coenzimi piridinici in spermatozoi. Atti $V^{\circ}$ Congresso Internazionale per la Riproduzione Animale e la Fecondazione Artificiale, 7, 475.

Bistocahi, M., D'Alessio, G. \& Leone, E. (1965) Pyridine coenzymes in epididymal spermatozoa. 2nd Meeting of Federation of European Biochemical Societies, A 124.

Bistocchi, M. \& Fetruccia, G. (1966) Ricerche sulla nucleotidopirofosfatasi seminale. Boll. Soc. ital. Biol. sper. 42, 1446.

Crotri, M. M. \& Kaplan, N. O. (1957) Procedures of determination of pyridine nucleotides. In: Methods in Enzymology, p. 890. Eds. S. P. Colowick and N. O. Kaplan. Academic Press, New York.

Gonse, P. H. (1962) Respiration and oxidative phosphorylation in relation to sperm motility. In: Spermatozoan Motility, p. 99. Ed. D. W. Bishop. American Association for the Advancement of Science, Washington, D.C.

Leone, E. \& Bonaduce, L. (1959) Soluble pyridine nucleotide nucleosidase from seminal vesicles. Biochim. biophys. Acta, 31, 292.

Leone, E. \& Santoranni, P. (1959) Enzimi e metabolismo del plasma seminale: il sistema nucleolitico seminale. Symp. Genetica et Biologica Italica, 9, 260.

ManN, T. (1948) Fructose and fructolysis in semen in relation to fertility. Lancet, i, 446.

MANN, T. (1964) The biochemistry of semen and of the male reproductive tract. Methuen, London.

VILLEE, C. A. (1962) An enzymic method for the assay of pyridine nucleotides in extracts of animal tissues. Biochem. 7. 83, 191.

WheAt, R. W., Krick, E. B. \& Brownlee, S. T. (1960) Nucleotide pyrophosphatase activities of seminal plasma. F. biol. Chem. 235, 3570 .

Winberg, H. (1941) The cozymase content of bird sperm. Ark. Kemi Miner. Geol. 15 B, 1. 